\title{
Docencia presencial, on-line o mixta: ¿Qué herramientas pedagógicas son mejor valoradas por el alumnado?
}

\author{
Daniel G. Palací López ${ }^{\mathrm{a}}$, Jesús Palací López ${ }^{\mathrm{b}}, \mathbf{M}^{\mathrm{a}}$ Isabel López Rodríguez $^{\mathrm{c}}$ \\ ${ }^{\text {a }}$ Production Support \& Continuous Improvement Department, International Flavors \& Fragrances \\ Inc, Benicarló, Spain, dapalpe@gmail.com ${ }^{\mathrm{b}}$ Universidad Rey Juan Carlos, Madrid, Spain, \\ jesus.palaci@urjc.es, ${ }^{\mathrm{c}}$ Dpto. de Economía Aplicada. Facultat d'Economia, Universitat de València, \\ Spain, maria.i.lopez@uv.es
}

\section{Resumen}

La puesta en marcha del proceso Bolonia en las universidades españolas, del que en el presente curso académico se cumple una década, ha ido abriendo las puertas a múltiples metodologías docentes en las que han tenido cabida numerosa Tecnologías de la Información y la Comunicación (TICs) y herramientas e-learning. Parece, por tanto, el momento de realizar un análisis del impacto que su uso ha tenido en el proceso de enseñanzaaprendizaje del alumnado. Atendiendo al resultado del mismo, se dispondrá de la información que permita seleccionar aquellas herramientas que, potencialmente, optimizarían los resultados del proceso mencionado. Una vez detectadas las que mejor resultado proporcionan, y atendiendo a su carácter presencial u on-line, se estaría en disposición de proponer las metodologías docentes que podrían obtener los mejores resultados ante tres posibles escenarios: docencia presencial, on-line o mixta.

Atendiendo a que las investigaciones en este campo han considerado, mayoritariamente, como output a optimizar el rendimiento académico, medido a través la calificación del estudiante, y con la finalidad de dar cabida a la opinión del mismo, en el presente trabajo se ha considera como output la percepción del alumnado, medida a través de la valoración que éste da a algunas herramientas pedagógicas. Concretamente se han seleccionado dos de ellas: una de carácter presencial (una Herramienta de Respuesta de Audiencia, H.R.A.) y otra e-learning (un contenedor hipermedia) que, lógicamente, no requiere de presencialidad. La recogida de datos que ha permitido el análisis se ha realizado a los alumnos de un grado ofertado por la Universitat de València, a través de un cuestionario ad-hoc. Los primeros resultados reflejan una mejor acogida a la H.R.A., con una mayor puntuación media (superior al 8), aunque la e-learning se postula útil para autoevaluarse. 
Docencia presencial, on-line o mixta: ¿Qué herramientas pedagógicas son mejor valoradas por el alumnado?

Palabras clave: e-learning, Herramienta de Respuesta de Audiencia, rendimiento académico, cuestionario ad-hoc, TICs

\begin{abstract}
The implementation of the Bologna process in Spanish universities, which has been active for a decade as of this academic year, facilitated the implementation of multiple teaching methodologies where numerous Information and Communication Technologies (ICTs) and e-learning tools have had a place. Thus, at this point, an analysis ought to be carried out of the impact that their use may have had on the teaching-learning process for the students. The results from such study will allow selecting the tools that could potentially optimize the results of the aforementioned process. Once the best ones among them have been identified, and taking into account their face-to-face or online nature, it will be posible to make a proposal of which teaching methodologies will lead to the best results in three possible scenarios: face-to-face, online or mixed teaching.
\end{abstract}

While most reaserach in this field focuses on the academic performance as the main output to optimize, measured through the student's qualification, the present work aims at taking the student's opinion into consideration. Therefore, the perception of the students with regards to some pedagogical tools is assessed in this work. Specifically, two tools have been selected: one face-to-face tool (an Audience Response Tool, H.R.A.) and another elearning one (a hypermedia container) that, logically, does not require attendance. The data collection required for its posterior analysis was carried out through an ad-hoc questionnaire presented to the students of a degree offered by the University of Valencia,. The first results reflect a better reception to H.R.A., with a higher average score (higher than 8), although elearning is postulated useful for self-evaluation.

Keywords: e-learning, Audience Response Tool, academic performance, adhoc questionnaire, ICTs.

\title{
1. Introducción
}

Parecía que, una década después de la puesta en marcha del plan Bolonia, era el momento de realizar una evaluación de la repercusión que éste había tenido en las universidades 
españolas. El enfoque desde el que hacerlo era amplio: valoración de la amplia oferta formativa, evaluación de la idoneidad de la estructura de los planes de estudio, percepción de los agentes involucrados, impacto del uso de las Tecnologías de la Información y la Comunicación (TICs) en el proceso de enseñanza-aprendizaje, etc. La situación de alarma sanitaria, derivada del COVID-19, decantó a los autores del presente trabajo hacia el análisis del impacto del uso de las TICs, pues ante la incertidumbre actual acerca de cómo se desarrollará la docencia en el curso venidero parece, cuanto menos, útil disponer de información que permita recomendar, en una indispensable planificación de la metodología docente (Calvo Bernardino \& Mingorance Arnáiz, 2013), el uso de unas TICs u otras frente a tres posibles escenarios: docencia presencial, on-line o mixta.

Así, el objetivo que se persigue es realizar un estudio comparativo de dos TIC: una Herramienta de respuesta de Audiencia (H.R.A.) de carácter presencial y otra e-learning (un contenedor hipermedia) que, lógicamente, no requiere presencialidad. Cabe aquí indicar que como en casi todas las TICs (López Rodríguez et al. 2015), el uso de las H.R.A. (Derek Bruff, 2009) han proporcionado hasta el momento buenos resultados en la docencia presencial (Calvo Roselló et al. 2017; Fuertes et al. 2016) y que el e-learning seleccionado (Martínez de Lejarza y Esparducer, 2010) ha sido diseñado teniendo en cuenta a los usuarios y posibles plataformas (Adell, 1995), lo que le ha posibilitado llegar de manera eficiente a todos los ususarios que estuviesen interesados en los contenidos del mismo. Sin embargo, casi todas las investigaciones que han permitido llegar a esas conclusiones no han tenido en cuenta la percepción/valoración del alumnado, agente de indiscutible importancia. Así, siguiendo algunos estudios recientes (López-Rodríguez \& Barac, 2019) el estudio comparativo se llevará a cabo contando con la opinion del alumno. Los datos que permitirán abordar dicho análisis corresponden a los alumnos de un grado ofertado por la Universitat de València y han sido recogidos a través de un cuestionario ad-hoc.

\section{Metodología}

Como es habitual en cualquier análisis de carácter exploratorio, la metodología utilizada será de tipo descriptivo, haciendo uso de las medidas de posición más relevantes (medidas de posición, medidas de dispersión, medidas de forma ...) y las representaciones gráficas pertientes que posibiliten tener una visión de la distribución de frecuencias de los datos así como la detección de datos anómalos, en el caso que los hubiera, y que podrían invalidar las conclusiones obtenidas. Concretamente, se hará un uso combinado de las gráficas de barras y del diagrama de Box Wiskher o de Caja-Bigotes. 
Docencia presencial, on-line o mixta: ¿Qué herramientas pedagógicas son mejor valoradas por el alumnado?

\section{Resultados}

En este epígrafe se expondrán los resultados obtenidos del estudio comparativo acerca de la percepción del alumnado sobre el uso de la H.R.A. y el e-learning. Concretamente:

- Se representarán númerica y gráficamente las distribuciones de las respuestas a las preguntas que hacían referencia a la aportación, que cada una de ellas tuvo desde el punto de vista de la comprensión de conceptos (a partir de aquí, $1^{\text {a }}$ pregunta) y la preparación del examen final de la asignatura (a partir de aquí, $2^{\mathrm{a}}$ pregunta).

- Se estudiarán las valoraciones que los estudantes dieron a ambas TICs, y se realizará un análisis comparativo que permita priorizar su uso, sin perder de vista el carácter de cada una de ellas (presencial y on-line, respectivamente). Concretamente se obtendrán las medidas de posición y diagrama de Caja-Bigotes de ambas valoraciones, tal y como se indicó en el epígrafe anterior.

Así, la tabla 1 corresponde a las representaciones numéricas de las distribuciones de frecuencias de las respuestas obtenidas a las preguntas $1^{\mathrm{a}}$ y $2^{\mathrm{a}}$ según la herramienta considerada sea la H.R.A. (presencial) o la e-earning (on-line)

Tabla 1. Distribución según ítems de la $1^{\mathrm{a}}$ pregunta, atendiendo a la TIC

\begin{tabular}{ccc|cc}
\hline \multicolumn{3}{c|}{ Ayuda a comprender conceptos } & \multicolumn{2}{c}{ Ayuda a preparar examen final } \\
\hline & H.R.A. & e-learning & H.R.A. & e-learning \\
\cline { 2 - 5 } No, en absoluto & $0.00 \%$ & $0.00 \%$ & $0.00 \%$ & $8.33 \%$ \\
Poco & $3.13 \%$ & $25.00 \%$ & $6.25 \%$ & $16.67 \%$ \\
Indiferente & $9.38 \%$ & $25.00 \%$ & $31.25 \%$ & $41.67 \%$ \\
Bastante & $53.13 \%$ & $41.67 \%$ & $40.63 \%$ & $33.33 \%$ \\
Mucho & $34.38 \%$ & $8.33 \%$ & $21.88 \%$ & $0.00 \%$ \\
\hline \multicolumn{4}{c}{ Fuente: Elaboración propia }
\end{tabular}

De cuya observación, puede deducirse que:

- El porcentaje de estudiantes que opina que el uso de la herramienta le ha ayudado a comprender mejor los conceptos de la asignatura (esto es, han seleccionado la opción Bastante o Mucho) asciende al 87.5\% en el caso de la H.R.A. y al $50 \%$ en el caso de la herramienta e-learning.

- Ese porcentaje resulta ser del $62.25 \%$ para la H.R.A. frente al $33.33 \%$ para la herramienta e-learning, cuando la cuestión planteada hace referencia a la ayuda percibida de cara a la preparación del examen final de la asignatura. 
Esto es, la H.R.A. se postula con mayor utilidad cuando se trata de comprender los diferentes conceptos de la materia así como ante la preparación de la prueba final. Conclusión que queda constatada a partir de la observación de las representaciones gráficas de las distribuciones consideradas (fig. 1).

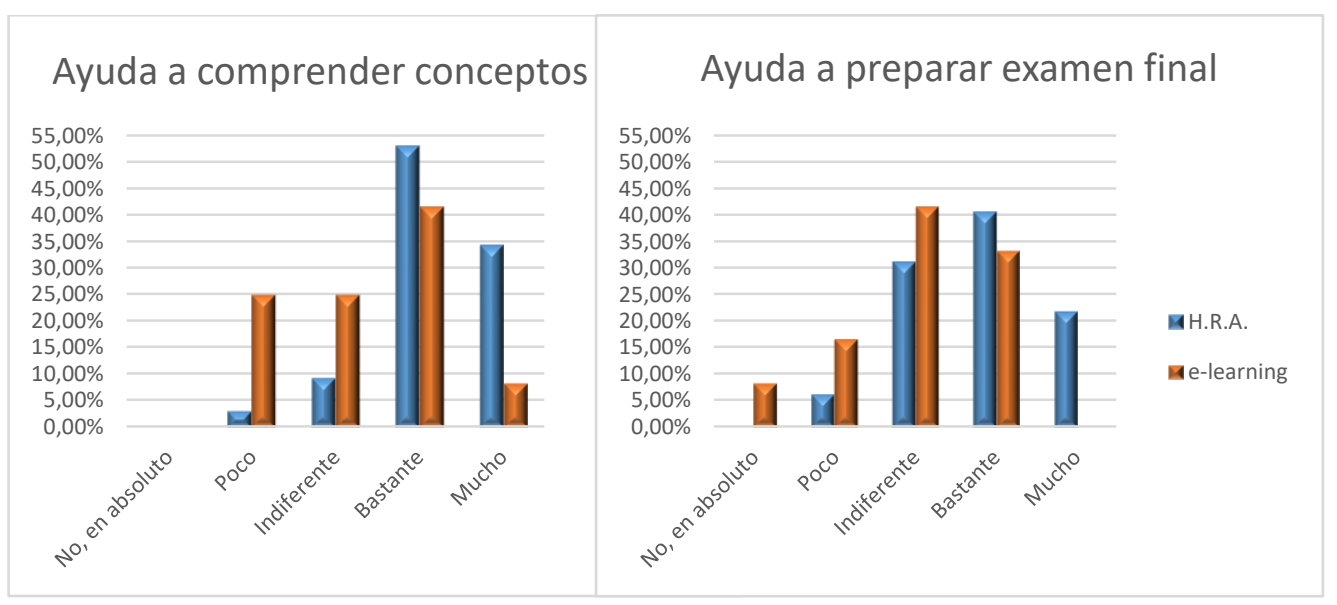

Fig. 1 Representación gráfica de la distribución de respuestas a preguntas $1^{a}$ y $2^{a}$, según TIC

Por otra parte, los datos contenidos en la tabla 2 (correspondientes a las medidas de posición de las valoraciones a ambas TICs):

Tabla 2. Medidas de posición de valoración otorgada por estudiantes, atendiendo a la TIC

\begin{tabular}{ccc}
\hline & Valoración (de 0 a 10) \\
Promedio & H.R.A. & e-learning \\
\cline { 2 - 3 } Desviación Estándar & 8.40625 & 7.33333 \\
Coeficiente de Variación de Pearson & 1.13192 & 1.66969 \\
Mediana & 0.1347 & 0.227 \\
Mínimo & 8 & 7.5 \\
Máximo & 6 & 4 \\
Rango & 10 & 10 \\
Sesgo Estandarizado & 4 & 6 \\
Curtosis Estandarizada & -0.74258 & -0.50082 \\
& Fuente: Elaboración propia & 0.00882 \\
\hline
\end{tabular}

Fuente: Elaboración propia 
Docencia presencial, on-line o mixta: ¿Qué herramientas pedagógicas son mejor valoradas por el alumnado?

Permiten concluir que:

- La TIC mejor valorada es la H.R.A. con una puntuación media de entorno al 8.4 (frente al 7.3333 del e-learning) y menor dispersión, atendiendo al valor del coeficiente de variación de Pearson ( 0.1347 frente a 0.227 ). Además, la puntuación más baja a la H.R.A. es un 6 , frente al 4 mínimo de la puntuación asignada al e-learning.

- Si se tiene en cuenta el valor de la mediana, al menos el 50\% de los estudiantes le asignan como mínimo un 8 a la H.R.A., siendo esa cota de un 7.5 para la herramienta elearning.

Por otra parte, atendiendo a los valores del sesgo y curtosis estandarizados se puede asumir la Normalidad para ambas variables (puntuaciones otorgadas por los estudiantes tanto a la H.R.A. como al e-learning). Sin embargo, el gráfico de caja-bigotes (fig.2) indica que hay un dato anómalo para la valoración de la H.R.A.

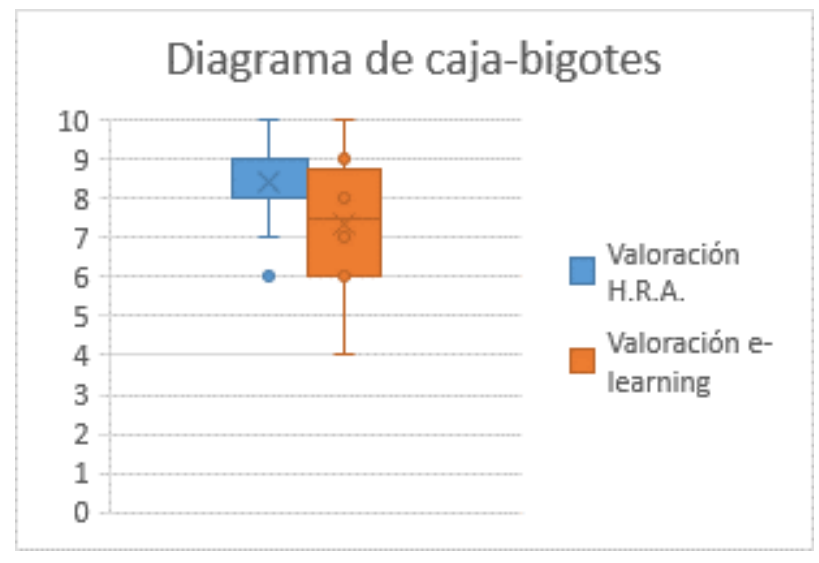

Fig. 2 Diagrama de Caja-Bigotes para ambas TICs

Tras la eliminación de dicho dato, se procede nuevamente a la obtención de las medidas de posición (tabla 3) : 
Tabla 3. Medidas de posición de valoración otorgada por estudiantes, atendiendo a la TIC

\begin{tabular}{ccc}
\hline \multicolumn{3}{c}{ Valoración (de 0 a 10) tres eliminación dato anómalo } \\
\cline { 2 - 3 } Promedio & H.R.A. & e-learning \\
\cline { 2 - 3 } Desviación Estándar & 8.56667 & 7.33333 \\
Coeficiente de Variación de Pearson & 0.9714 & 1.66969 \\
Mediana & 0.1134 & 0.2277 \\
Mínimo & 8.5 & 7.5 \\
Máximo & 7 & 4 \\
Rango & 10 & 10 \\
Sesgo Estandarizado & 3 & 6 \\
Curtosis Estandarizada & 0.09172 & -0.50082 \\
& -1.0219 & 0.00882213 \\
\hline
\end{tabular}

Fuente: Elaboración propia

Obteniéndose, a partir de los valores de los parámetros conclusiones similares, esto es, la H.R.A. resulta mejor valorada (mayor puntuación media, menor variabilidad, con mayor puntuación mínimar, con un valor de la mediana superior a la herramienta e-learning). Por otra parte, sigue pudiendo asumirse la Normalidad para las puntuaciones otorgadas por los estudiantes a ambas TICs. En cualquier caso, cabe destacar que la herramienta e-learning también recibió una muy buena aceptación por parte del alumnado. Se puede, en este sentido, recoger a modo de ejemplo el siguiente comentario, expuesto por los estudiantes en el cuestionario: "Buena para comprobar resultados", haciendo referencia a las cuestiones y problemas de autoevaluación.

\section{Conclusiones}

Teniendo en cuenta que el objetivo propuesto, en el trabajo que se presenta, era realizar un estudio del impacto percibido, en el proceso de enseñanza-aprendizaje, por el alumnado acerca del uso de dos TICs diferenciadas, principalmente, por su carácter presencial y online (una H.R.A. y una herramienta e-learning), puede concluirse que aunque ambas han contado con una aceptación más que satisfactoria tanto a la hora de asimilar los diferentes conceptos que componían la materia como a la de preparar su evaluación, la H.R.A. ha resultado más útil para el alumnado, que en un porcentaje superior al $62 \%$ ha contestado que la herramienta les ha servido "Bastante" o "Mucho" para los fines propuestos, porcentaje que no ha sido superior al $50 \%$ en el caso de la e-learning. También en la 
Docencia presencial, on-line o mixta: ¿Qué herramientas pedagógicas son mejor valoradas por el alumnado?

puntuación otorgada, la H.R.A. se posiciona por delante con mayor valoración media (8.57 frente a 7.33) y además, todos los estudiantes le han proporcionado una puntuación mínima de notable (7). Sin embargo la herramienta e-learning se postula muy útil para la autoevaluación del alumnado. De todo lo expuesto podría, por tanto, proponerse el uso combinado de ambas TICs en una metodología docente dirigida a una docencia presencial o mixta, haciendo más enfásis en la utilización de la H.R.A. y si la docencia fuese on-line, a tenor de los buenos resultados de la herramienta e-learning y la imposibilidad material del uso de la H.R.A., la propuesta iría claramente encaminada al uso de la e-learning.

\section{Agradecimientos}

Expresamos nuestro agradecimiento al Servei de Formació Permanent i Innovació Educativa así como a los Vicerrectorats d'Estudis de Grau i Política Lingüística i de Polítiques de Formació i Qualitat Educativa de la Universitat de València por la concesión del proyecto de innovación educativa UV-SFPIE_PID19-1095774 que ha financiado parcialmente esta comunicación.

\section{Referencias}

Adell, Jordi (1995). La navegación hipertextual en el World Wide Web: implicaciones para el diseño de materiales educativos. Palma de Malorca. EDUTEC'95. Recuperado el 22/05/2019 de http://nti.uji.es/docs/nti/edutec95.html

Calvo Bernardino, A. \& Mingorance Arnáiz, A.C. (2013). Planificación de la metodología docente adaptada al EEES: una propuesta en el ámbito de la economía Aplicada, Revista complutense de la educación, vol. 24, $\mathrm{n}^{\mathrm{o}}$ 1, pp. 185-210.

Calvo Roselló, V.; López Rodríguez, M.I.; Ruiz Ponce, F. (2017). Uso de un Electronic Voting System: una radiografía del aula universitaria en tiempo real. 5th International Conference on Innovation, Documentation and Teaching Technologies (INNODOCT 2017), ISBN: 978-849048-612-2, pp. 715-726. Valencia.

Derek Bruff, D. (2009). Teaching with classroom response systems: creating active learning environments. Ed. Wiley.

Fuertes, A., García, M., Castaño, M.A., López, E., Zacares, M., Cobos, M., Ferris, R., Grimaldo, F. (2016). Uso de herramientas de respuesta de audiencia en la docencia presencial universitaria. Un primer contacto. En: Actas de las XXII Jenui. Almería, Spain, 261-268.

López Rodríguez, M.I.; Palací López, J.; Palací López, D. (2015). Use of ICTs in degree studies: a descriptive analysis. Sevilla. ICERI 15. IATED, pp. 2286-2290.

López-Rodríguez, M. I., y Barac. M. (2019). Valoración del alumnado sobre el uso de Clickers y vídeo tutoriales en educación superior. Research in Education and Learning Innovation Archives, 22, 29-44. DOI: 10.7203/realia.22.14582 
Martínez de Lejarza y Esparducer, J. (2010). Contenedor hipermedia de Estadística Aplicada a la Economía y Ciencias Sociales (CEACES): una aproximación. @tic. Revista d'innovació educative, 5, 77-82 Recuperado el $22 / 05 / 2019$ de http://ojs.uv.es/index.php/attic/article/view/198 